\title{
Assessing the severity of valve stenosis
}

\author{
O ODEMUYIWA, R J C HALL \\ From the Department of Cardiology, Royal Victoria Infirmary, Newcastle upon Tyne
}

In the early days of cardiac catheterisation the pressure gradient was the main criterion for the estimation of the severity of valve stenosis. The gradient alone, however, is not a true reflection of stenosis since it is heavily dependent on the cardiac output and heart rate. Thus its use can lead to gross errors in the diagnosis of those severe valve lesions that considerably reduce cardiac output and hence produce only a small gradient. This situation is not uncommon in end stage aortic stenosis, a condition in which correct diagnosis is essential because surgery can be life saving. ${ }^{1}$ Increases of left ventricular end diastolic pressure can confound mitral valve gradient measurements and also lead to underestimation of severity even by experienced investigators. ${ }^{2}$ A major advance in the assessment of valve stenosis came in 1951 when Gorlin and Gorlin derived the formula which related heart rate, pressure gradient, and cardiac output to valve area. ${ }^{3}$ Several sources of inaccuracy became apparent as use of this formula increased. The hydraulic equations from which the Gorlin formula was derived assume flow through an idealised orifice and not through the funnel shaped spout of a stenotic mitral valve. There are also theoretical deficiencies in the use of the square root of the mean pressure gradient instead of the mean square root of the instantaneous pressure gradients. ${ }^{4}$ The formula depends on the accurate measurement of cardiac output, but each application of the Fick principle to this measurement has its unique sources of imprecision. Accurate pressure measi:-ements are also important, and significant errors cai be introduced first in the measurement of the pulmonary artery wedge pressure itself and alse in its use as ? substitute for left atrial pressure. ${ }^{5}$ The Goriin formula is also misleading in the presence of important regurgitation. Lastly the formula was verified by dizital palpation at operation or in necropsy specimens neither of which could accurately represent valve dimeñsions in life.

Requests for reprints to D: R I C Hall, Department of Cardiology, Royal Victoria Infirmary, Queen Victuria Road, Newcastle upon Tyne NE1 4LP.
The sources of inaccuracy in the Gorlin formula have been tackled either by direct measurements or indirectly by a mathematical approach. Valve areas have been measured directly with templates or sizers. ${ }^{6}$ These have been quite successful with the mitral valve, although there is uncertainty about the change in shape and size of an excised mitral valve without its subvalvar apparatus. Aortic valves are more difficult because they tend to be forced open, fragment, or crack during sizing. The indirect mathematical approach is to try to derive new and more accurate equations based on solid theoretical foundations and more certain corroboration. A recent equation ${ }^{7}$ eliminates the diastolic filling period and uses a new constant, but it remains to be seen whether these will compensate for the limitations of measuring cardiac output and pressure.

Although the validity of catheter data has largely been accepted without question it is apparent that the assumptions which underpin correlative studies are not true. The components of the so-called gold standard are themselves not made of gold and the quality of the standard must vary both between and within centres. Despite these limitations cardiac catheterisation has been central to the increasing understanding of the pathophysiology of cardiovascular disease, and by serving as a reasonably reliable reference has spurred improvements in cardiac surgery and the interpretation of physical signs. Cardiac catheterisation is however expensive, time consuming, and sometimes dangerous, ${ }^{8}$ and therefore as non-invasive techniques have improved the focus has shifted to their use in assessing valve stenosis. These techniques usually evoke an initial enthusiasm that is followed by disappointment until some middle ground is found between these extremes.

Echocardiography is the most useful and thoroughly investigated of the non-invasive techniques. Early work on mitral stenosis examined particular variables in relation to findings at cardiac catheterisation or operation, and indices of severity such as the diastolic closure rate, ${ }^{9}$ mitral valve closure index, ${ }^{10}$ and changes in left ventricular 
dimensions ${ }^{1112}$ were derived. These indices went through the usual sequence of evaluation and detailed study ${ }^{13}$ and were often the subject of acrimonious debate. In some eyes these and other studies like them confirmed the echocardiogram as a potential replacement for cardiac catheterisation, ${ }^{14}$ but others believe that the catheter laboratory is still the only source of accurate information. ${ }^{15}$ It was not clear how often the echocardiogram provided information not available from the clinical examination and simple tests (such as the electrocardiogram and chest $x$ ray) and made a contribution to decisions about operation without catheterisation. This is important because as non-invasive techniques proliferate there is a tendency for patients to undergo an increasing number of investigations before the inevitable ritual of catheterisation. One approach has been to simulate the diagnostic cascade. ${ }^{16-18}$ It is estimated that about $70 \%$ of patients investigated for valve surgery may not need catheterisation because mild and severe disease could be accurately defined non-invasively. These studies also highlighted some of the difficulties such as the tendency for the echocardiogram to overestimate aortic stenois ${ }^{18}$ and the limitations of echocardiographic assessment of moderately severe mixed or multiple lesions. This latter category has proved the most difficult to assess by any of the current techniques including catheterisation because the definition of this group is still largely subjective. ${ }^{18}$

Along with developments in non-invasive cardiology has grown the inclination to derive new equations that aim to improve assessment by incorporating several variables in much the same way as the Gorlin formula. Such equations are based on the assumption of a precise, usually linear, relation between two or more physiological variables in a patient population. These relations may be applicable to group data, but there is a normal distribution of both physiological variables and their responses to other stimuli that may introduce doubt about applying them to individual patients. Such equations are often an oversimplification of the complex interplay of these physiological and statistical considerations. Bennett's formula which is one such equation ${ }^{19}$ assumes that since left ventricular wall stress is directly related to intracavitary pressure the degree of adaptive hypertrophy required to normalise this stress can be used to calculate the left ventricular systolic pressure. On page 155 of this issue Dancy has re-examined Bennett's formula for the estimation of the peak pressure gradient in aortic stenosis and has arrived at two main conclusions. ${ }^{20}$ The first obvious but often neglected point is that the quality of the echocardiogram is of paramount importance. In the $42 \%$ of Dancy's adult patients who had very high quality echocardiograms the severity of aortic stenosis correlated more strongly with direct measurements of left ventricular wall thickness than with left ventricular mass or pressure derived from Bennett's equation. Echocardiograms of less than this quality were no better than the electrocardiograms in assessing aortic stenosis and their value for predicting the severity of aortic stenosis was not improved by use of the Bennett formula. The second conclusion emphasises the fact that small errors even in the most careful measurements can be magnified several fold if variables are derived from formulas containing complex mathematical functions. A difference of as little as $1 \mathrm{~mm}$ in left ventricular dimensions changed calculated left ventricular pressure by $20 \mathrm{~mm} \mathrm{Hg}$.

Dancy's work highlights some of the basic dilemmas encountered when mediocre non-invasive data are compared with inaccurate catheter or surgical data. Image quality is relatively easy to define; less definite but probably as important are the skill, care, and experience that among other imponderables constitute the "quality" of noninvasive and catheter data. The weakness of correlative studies that include such data is therefore obvious. These studies often use independent observers to reduce bias but then reintroduce inconsistency in settling differences of opinion by the doubtful concept of consensus. This may provide coefficients of variation which are sound in scientific terms for group data but are probably of little relevance to the clinician and the individual patient. It follows that most of the assumptions about accuracy are illusory. In making decisions the clinician neèds to know how much value to place on a particular measurement and therefore it is the $95 \%$ confidence limits that are important. A variable with a relatively narrow confidence limit is more useful in distinguishing normal values from abnormal ones and in grading severity of disease than one with wider limits. One way of establishing these limits is to study the reproducibility of these measurements ${ }^{21} 22$ and echocardiography is ideal in this respect because it can be repeated many times without risk to the patients. Doppler echocardiography also needs to be studied in this way as it is still in its excellent correlative phase ${ }^{23} 24$ and its limitations are yet to be established. The reproducibility of the catheter data against which non-invasive data are compared has been virtually unchallenged because the technique does not lend itself to this approach. Reproducibility studies will need to be performed in each centre and reviewed as experience is gained in using the technique. The emphasis must be on striving for high quality images and poor data should be discarded.

More studies simulating the diagnostic cascade are 
required to identify the relative merits of these investigations and thus avoid tedious, expensive, and time consuming duplication. Non-invasive techniques can then be used not as a wholesale replacement for cardiac catheterisation but as a substitute in cases where they establish the diagnostic and therapeutic situation beyond doubt.

\section{References}

1 Morgan D, Hall RJC. Occult aortic stenosis as cause of intractable heart failure. $\mathrm{Br} \mathrm{Med} \mathcal{F} 1979$; i: 784-7.

2 Traill TA, St John Sutton MG, Gibson DG. Mitral stenosis with high left ventricular diastolic pressure. $\mathrm{Br}$ Heart F 1979; 41: 405-11.

3 Gorlin R, Gorlin S. Hydraulic formula for calculation of the area of the stenotic mitral valve, other cardiac valves and central circulatory shunts. Am Heart $\mathcal{F} 1951$; 41: 1-28.

4 Rodrigo FA, Snellen HA. Estimation of valve area and "valvular resistance". Am Heart f 1953; 45: 1-12.

5 Hosenpud J, McAnulty J, Morton M. Overestimation of mitral valve gradients obtained by phasic pulmonary capillary wedge pressure. Cathet Cardiovasc Diagn 1983; 9: 283-90.

6 Marino P, Zanolla L, Perini G, Salazzari G, Conti F, Poppi A. Critical assessment of two dimensional echocardiographic estimation of mitral valve area in rheumatic mitral valve disease. Calcific deposits in the valve as a major determinant of the accuracy of the method. Eur Heart $\mathcal{F}$ 1981; 2: 197-203.

7 Seitz W, Marino P, Zanolla L, Buonanno C, Mcllroy M, Spiel M. Cardiac valve orifice equations independent of valvular flow intervals: application to mitral valve area computation in mitral stenosis and comparison with the Gorlin formula and direct anatomical measurements. Cardiovasc Res 1984; 18: 669-74.

8 Karnegis J, Heinz J. The risk of diagnostic cardiovascular catheterisation. Am Heart 7 1979; 97: 291-7.

9 Edler I. Ultrasound cardiography in mitral valve stenosis. Am $\mathcal{f}$ Cardiol 1967; 19: 18-31.

10 Shiu MF. Mitral valve closure index. Echocardiographic index of severity of mitral stenosis. $\mathrm{Br}$ Heart F 1977; 39: 839-43.

11 Gibson DG, Brown D. Measurement of instantaneous left ventricular dimension and filling rate in man, using echocardiography. Br Heart $\mathcal{f} 1973$; 35: 1141-9.

12 Hall RJC, Clarke SE, Brown D. Evaluation of posterior aortic wall echogram in diagnosis of mitral valve disease. Br Heart f 1979; 41: 522-8.

13 Hall R, Austin A, Hunter S. M-mode echogram as a means of distinguishing between mild and severe mitral stenosis. Br Heart F 1981; 46: 486-91.

14 Motro M, Neufeld H. Should patients with pure mitral stenosis undergo cardiac catheterisation? Am $\mathcal{f}$ Cardiol 1980; 46: 515-6.

15 Roberts C. Reasons for cardiac catheterisation before cardiac valve replacement. $N$ Engl $f$ Med 1982; 306: 1291-3.

16 Alpert J, Lawrence S, Cohn P, Grossman W. The diagnostic accuracy of combined clinical and non-invasive cardiac evaluation. Comparison with findings at cardiac catheterisation. Cathet Cardiovasc Diagn 1980; 6: 359-70.

17 St John Sutton G, St John Sutton M, Oldershaw P, et al. Valve replacement without pre-operative cardiac catheterisation. $N$ Engl $f$ Med 1981; 305: 1233-8.

18 Hall RJC, Kadushi OA, Evemy K. Need for cardiac catheterisation in assessment of patients for valve surgery. Br Heart $\mathcal{F}$ 1983; 49: 268-75.

19 Bennett DH, Evans DW, Raj MVJ. Echocardiographic left ventricular dimensions in pressure and volume overload. Their use in assessing aortic stenosis. $\mathrm{Br}$ Heart $\mathcal{f}$ 1975; 37: 971-7.

20 Dancy M. Comparison of electrocardiographic and echocardiographic measures of left ventricular hypertrophy in the assessment of aortic stenosis. Br Heart $\mathcal{f}$ 1986; 55: 155-61.

21 Martin R, Rakowski H, Kleiman J, Beaver W, London E, Popp R. Reliability and reproducibility of two dimensional echocardiographic measurement of the stenotic mitral valve orifice area. Am f Cardiol 1979; 43: 560-8.

22 Bullock R, Appleton D, Griffiths C, Albers C, Amer H, Hall RJC. Precision of digitized M-mode echocardiogram for clinical practice. Eur Heart $\mathcal{F}$ 1984; 5: 941-7.

23 Holen J, Rashid R, Landmark K, Simonsen S. Determination of pressure gradient in mitral stenosis with a non-invasive ultrasound Doppler technique. Acta Med Scand 1976; 199: 455-60.

24 Hatle L, Angelsen A, Tromsdal A. Non-invasive assessment of aortic stenosis by Doppler ultrasound. $\mathrm{Br}$ Heart $\mathcal{F}$ 1980; 43: 284-92. 